\title{
Fatigue Fracture Behavior of Solid-State Extruded High-Density Polyethylene
}

\author{
Norihiro KaIYA, Atsushi Takahara, and Tisato KaJIYAMA* \\ Department of Applied Chemistry, Faculty of Engineering, Kyushu University, \\ Hakozaki, Higashi-ku, Fukuoka 812, Japan
}

(Received January 12, 1989)

\begin{abstract}
High-density polyethylene (HDPE) specimens for fatigue experiments were prepared by solid-state extrusion. Variations of dynamic viscoelasticity and surface temperature of the specimen were measured under cyclic fatigue. Fatigue behavior of extruded HDPE showed characteristic features depending on the type of cyclic fatigue. In the case of the fatigue test including compression deformation, extruded HDPE fatigue-fractured via the formation of a kinkband along the maximum shear stress direction. On the other hand, in the case of the pure tension fatigue test, the fatigue fracture surface generated in the normal direction of cyclic straining. The total hysteresis loss energy is divided into the two parts: the energy consumed to fracture the specimen and the energy dissipated as heat under cyclic fatigue. A fatigue fracture criterion based on the magnitude of the hysteresis loss energy consumed for a structural change was established. The fatigue strength of extruded HDPE estimated on the basis of this criterion was high in the cases of pure tension and pure compression fatigue tests, and also, was low in that of the tensioncompression fatigue test.
\end{abstract}

KEY WORDS Fatigue Behavior / Oriented HDPE / Fatigue Fracture Criterion / Heat Generation Under Cyclic Fatigue / Kink-Band /

Since linear polymers have strong covalent bonds along their main chains, they exhibit high strength and stiffness along the direction of the molecular axes. Specimens with high strength have been prepared from various polymers by various techniques. ${ }^{1}$ These materials are highly oriented with high tenacity in the direction of processing. Such highly oriented polymeric materials possess a high modulus equal to that of metalic materials. It is very important with respect to reliability for engineering applications to clarify the fatigue behavior of such polymeric materials.

Studies on heat generation under cyclic fatigue were performed on the basis of the viscoelastic characteristics of polymeric materials. $^{2-4}$ Recently, in our laboratory, the fatigue behavior of various polymeric ma- terials was analysed on the basis of continuous measurements of dynamic viscoelasticity during fatigue process. ${ }^{5-8}$ From these dynamic viscoelastic investigations, two characteristic types of fatigue failure, brittle and thermal failures, were classified depending on factors such as the fatigue conditions for each strain amplitude, the magnitude of heat transfer coefficient and environmental temperature. The fatigue fracture criterion was established on the basis of average energy loss during fatigue process.

When highly oriented polymers were deformed with compressive stress along the orientation axis, the formation of kink-bands was generally observed..$^{9-11}$ Robertson revealed that kink-bands often developed, resulting in a change of crystalline orientation, when the

* To whom correspondence should be addressed. 
shear stress inclined by certain degrees to the orientation axis is imposed to the drawn highdensity polyethylene film. ${ }^{9}$ From the results of wide-angle $\mathrm{X}$-ray diffraction measurements, he concluded that the structural changes within the kink-bands were caused from crystal flattening and crystal twinning or pseudotwinning on planes intersecting the molecular axis.

Shigematsu et al. observed that lamellar crystals and amorphous layers were stacked alternatively along the extruding direction of the solid-state extrudates of high-density polyethylene and that kink-bands were formed by compressing the specimens along the extrusion direction. ${ }^{10,11}$ They revealed that the fiber axis was inclined from the original axial direction by $70-75^{\circ}$, and also, that the lamellar crystals were inclined to the fiber axis by $55-60^{\circ}$ in the kink-band and stacked parallel to the kink boundary from wide and smallangle X-ray diffraction studies and electron microscopic observations. They proposed that the super-structural changes during the formation of the kink-bands could be well explained in terms of the uniform $c$-axis shear deformation of the crystallites combined with the intercrystallite slip, being accompanied by rotation of fiber axes of the crystallite blocks.

Since the formation of kink-bands may reduce fatigue strength, it is important to analyse the fatigue behavior of oriented polymers under cyclic straining with a compression component. In this study, the fatigue behavior of solid-state extrudate of high-density polyethylene under various types of cyclic deformation was investigated by continuous measurements of dynamic viscoelasticity and surface temperature during cyclic straining. Also, the fatigue fracture criterion based on the hysteresis loss was established to estimate the magnitude of the fatigue lifetime of the highly oriented solid-state extrudate of high-density polyethylene.

\section{EXPERIMENTAL}

\section{Specimen Preparation}

The polymer used was high-density polyethylene (Hizex 1300J, Mitsui Petrochemical Co., Ltd.) with a melt index of 14.0. The billets for the extruding experiment were cut from a thick sheet prepared by compression molding. The diameter of the billet was $10 \mathrm{~mm}$ and the length from 20 to $30 \mathrm{~mm}$. The extruding experiment was carried out in a manner similar to that in previous reports. ${ }^{8,12}$ The extruding temperature was $353 \mathrm{~K}$ and corresponded to that of the mechanical crystalline relaxation range of HDPE. ${ }^{13,14}$ The die diameter of the entrance, $2 R$, was $10 \mathrm{~mm}$ and that of the exit, $2 r$, was $3 \mathrm{~mm}$. The extrusion ratio, $\lambda$, corresponding to the draw ratio was defined as the ratio of the cross sectional areas of the extrudate before and after extrusion, $\lambda=(R / r)^{2}$. The extrusion ratio of extruded HDPE was 11.1 .

\section{Fatigue Testing}

Figure 1 shows a block diagram of the homemade fatigue tester ${ }^{5,6}$ used in this study. Dynamic viscoelasticity during fatigue process can be measured by the fatigue tester. A pyroelectric infrared radiation thermometer (ER-2008 SD, Matsushita Electric Co., Ltd.) was used to detect surface temperature rise of a specimen. The minimum diameter of the area to be measured was $2 \mathrm{~mm}$ when the distance

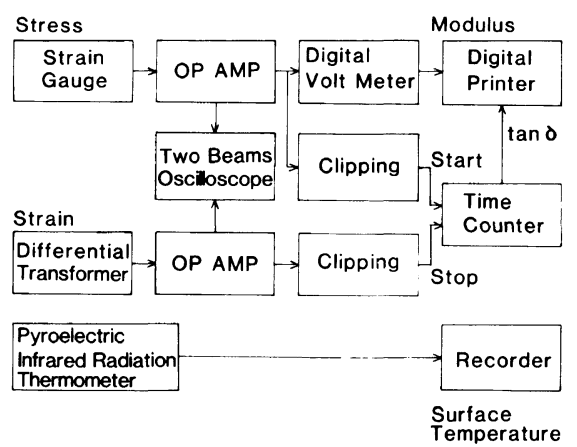

Figure 1. Block diagram of the measuring system of fatigue tester. 
between the thermometer and the specimen was $140 \mathrm{~mm}$. The output from the thermometer was continuously recorded on a recorder.

Fatigue tests were carried out at a frequency of $6.91 \mathrm{~Hz}$ and ambient temperature of $303 \mathrm{~K}$. As shown in Figure 2, the three modes of cyclic straining: a) tension type, b) tensioncompression type, and c) compression type were used. In the case of the tension type fatigue, the minimum sinusoidal strain, $\varepsilon_{\min }$,

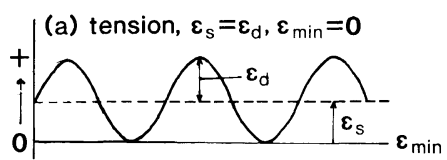

(b) tension-compression, $\varepsilon_{\mathrm{s}}=\mathbf{0}$

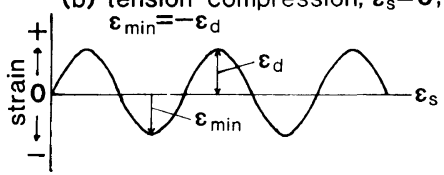

(c) compression, $\varepsilon_{\mathrm{s}}=-\boldsymbol{\varepsilon}_{\mathrm{d}}$,

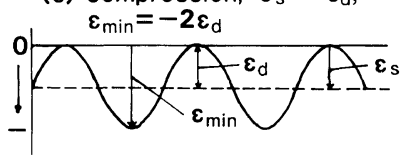

Figure 2. Strain variables for three types of fatigue tests: a), tension type; b) tension-compression type; c) compression type. was 0 and the magnitude of the static strain, $\varepsilon_{\mathrm{s}}$, was equal to that of the dynamic strain amplitude, $\varepsilon_{\mathrm{d}}$. By this mode, the elongation of a specimen is caused only by tensile force. Since the orientation factor of the specimen used in this study is 0.99 , the creep deformation of the specimen is negligible during cyclic tensile straining. In the case of tension-compression type fatigue, the same magnitude of tension and compression strains on the zero static strain were applied to a specimen, $\varepsilon_{\min }=-\varepsilon_{\mathrm{d}}$. In the case of compression type fatigue, the maximum sinusoidal strain was 0 and the magnitude of the minimum strain was two times that of the dynamic strain amplitude, $\varepsilon_{\min }=-2 \varepsilon_{\mathrm{d}}$.

\section{Morphological Observation of Fatigue-Frac- tured Specimen \\ Scanning electron microscope (SEM) (S- 430, Hitachi, Co., Ltd.) was used for mor- phological observation of extruded HDPE samples after fatigue failure.}

\section{RESULTS AND DISCUSSION}

\section{Morphological Observation}

Figure 3 shows a photograph of fatigue

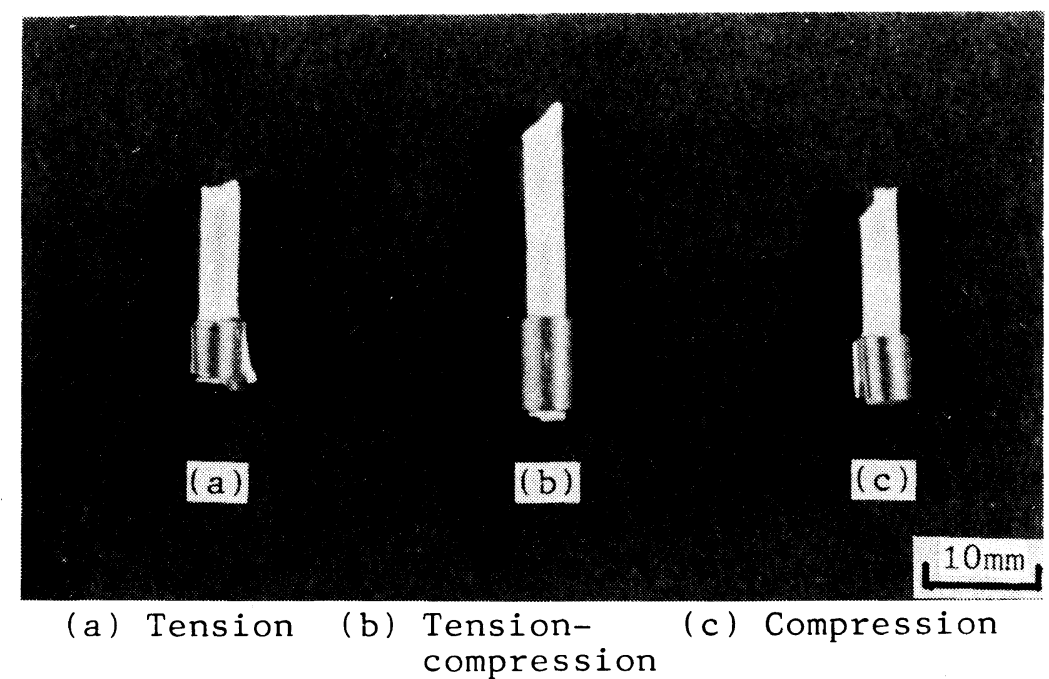

Figure 3. Photograph of fatigue fractured specimens under various cyclic straining: a), tension type; b), tension-compression type; c) compression type. The scale bar indicates $10 \mathrm{~mm}$. 


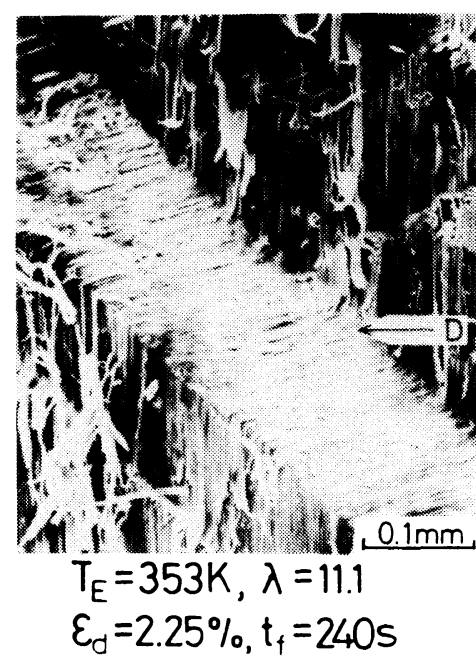

(a)

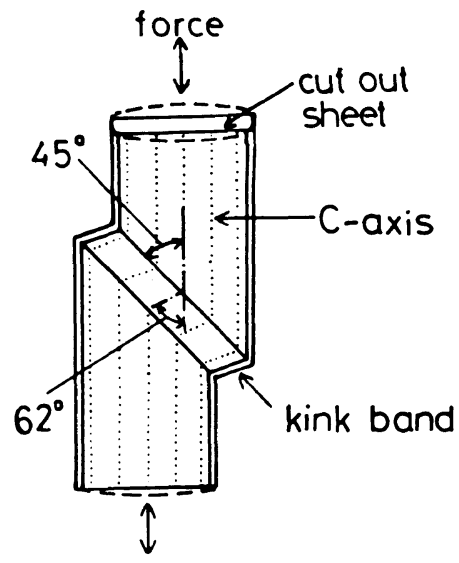

(b)

Figure 4. Scanning electron micrograph for fatigued extrudate under tension-compression type cyclic straining. The portion " $D$ " indicates a kink-band. The sample for SEM observation was cut out in liquid $\mathrm{N}_{2}$ as represented in (b).

fractured specimens for the three different modes of cyclic straining. The fatigue fracture surfaces exhibited characteristic shapes depending on the mode of cyclic straining. The fatigue fracture surface under tension type fatigue (a) appeared normal to the direction of cyclic straining. On the other hand, under tension-compression or compression type fatigue, the fracture surface inclined by $45^{\circ}$ to the direction of cyclic straining. This inclination of the fatigue fracture surface was due to the formation of a kink-band along the direction of the maximum shear stress under cyclic straining as shown in Figure 4.

Figure 4(a) is a scanning electron micrograph of a kink-band formed inside the specimen in the case of fatigue test imposing cyclic straining with a compression component. The sheet-shaped sample for SEM observation was cut out in liquid $\mathrm{N}_{2}$ from the compressed cylindrical specimen as shown in Figure 4(b). The kink-band was formed along the $45^{\circ}$ line to the compression direction parallel to the $c$ axis. On the boundary of the kink-band, the $c$ axis of HDPE chain bent sharply by $62^{\circ}$ to the extruding direction.

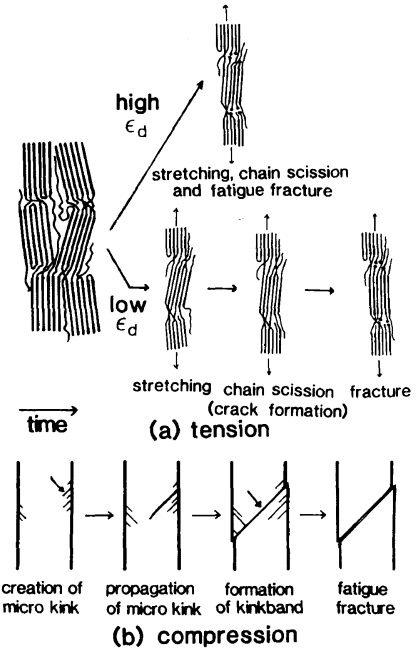

Figure 5. Schematic representation of fatigue fracture mechanisms for extruded HDPE under a) tension type and b) compression type cyclic straining.

Figure 5 shows a schematic representation of fatigue fracture processes of highly oriented HDPE under tension type (a) and compression type (b) fatigues. The original specimens are composed of both lamellar crystals and amorphous layers stacked alternatively along the extruding direction. The long-periodical struc- 
ture was clarified from studies based on small angle X-ray scattering. In the case of tension type fatigue, the cracks might initiate from chain scissions in the amorphous or interfacial region and propagate along the boundary between lamellar crystals. Thus, the fatigue fracture surface under tension type fatigue is produced perpendicular to the cyclic tensile straining (extruding direction). On the other hand, in the case of compression type fatigue, kinkbands are formed along the direction of the maximum shear stress under compression cycle straining. Since fibrils or molecular chains are sharply bent from their original direction on the boundary of the kink-band as shown in Figure 4, it is reasonably considered that many crazes are generated along this boundary under cyclic straining. Then, the cracks propagate and grow along the boundary of kinkband. Therefore, the fatigue fracture surface under compression type fatigue inclines by $45^{\circ}$ to the direction of cyclic straining.

\section{Relationship between Dynamic Strain Ampli- tude and Fatigue Lifetime}

Figure 6 shows the relationship between dynamic strain amplitude, $\varepsilon_{\mathrm{d}}$, and fatigue lifetime for extruded HDPEs which were fatigue-tested by three different strain modes. Comparing these fatigue characteristics at the same magnitude of strain amplitude, the fa-

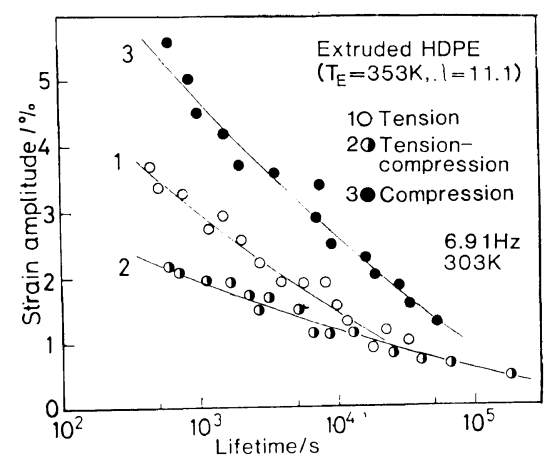

Figure 6. Relationships between imposed strain amplitude and fatigue lifetime for the extruded HDPE under various cyclic straining conditions. tigue lifetime of extruded HDPE under tension-compression type fatigue was shorter than those under the other two types of cyclic straining. It is reasonable that the shortest fatigue lifetime in the case of tensioncompression type fatigue test may be attributed to the formation of kink-bands during compression deformation and also, extension of the kink-band boundary region during tensile deformation, resulting in the propagation of cracks along the kink-band boundary. On the other hand, in the case of pure compression type fatigue, the formed kink-band absorbs the cyclic compression energy without expanding crazes or cracks generated in the kink-band boundary. Therefore, the fatigue lifetime under compression type fatigue becomes longest compared with the other two cases.

Variation of Dynamic Viscoelasticity and Surface Temperature during Cyclic Fatigue Straining

Figures 7-9 show variations of the dynamic storage modulus, $E^{\prime}$, the mechanical loss tangent, $\tan \delta$, and the surface temperature rise, $\theta_{\mathrm{s}}\left(=T_{\mathrm{s}}-T_{0}\right.$, where $T_{\mathrm{s}}$ and $T_{0}$ are the

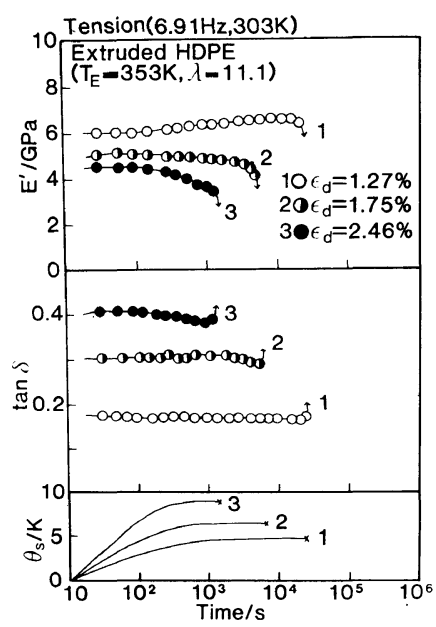

Figure 7. Variation of dynamic storage modulus, $E^{\prime}$, mechanical loss tangent, $\tan \delta$ and surface temperature, $\theta_{\mathrm{s}}$ for the extruded HDPE with time during the fatigue process.under tension type fatigue. 
surface temperature and the ambient temperature, respectively.) with fatigue time in three different cyclic straining modes. In all fatigue tests, the magnitude of $E^{\prime}$ decreased and that of $\tan \delta$ increased with the magnitude of the imposed strain amplitude. This was due to the contribution of non-linear viscoelasticity. 5,6

In the case of tension type fatigue under small imposed strain amplitude (Figure 7), the magnitude of $E^{\prime}$ gradually increased during fatigue process. Maximum of $E^{\prime}$ and minimum of $\tan \delta$ were observed on approaching the point of failure. This is typical behavior of brittle failure observed for unoriented samples. ${ }^{5,6}$ Maximum of $E^{\prime}$ and minimum of $\tan \delta$ at the last stage of fatigue process indicate considerable contribution of elastic term, such as orientation of molecular chains under cyclic fatigue. Maximum of $E^{\prime}$ and minimum of $\tan \delta$ gradually disappeared with increase in the magnitude of imposed strain amplitude. At a large imposed strain amplitude, $E^{\prime}$ decreased on approaching the point of the fatigue failure. The magnitude of $\theta_{\mathrm{s}}$ increased within a few hundred seconds of the initial stage of fatigue process and approached equilibrium between heat generation and heat dissipation.

In the case of tension-compression type fatigue under small imposed strain amplitude (Figure 8), the magnitudes of $E^{\prime}$ and $\tan \delta$ did not remarkably change during fatigue process. The slight maximum of $E^{\prime}$ and minimum of $\tan \delta$ were observed on approaching the point of failure for the all cases studied here. The maximum of $E^{\prime}$ and minimum of $\tan \delta$ gradually disappeared with an increase in the magnitude of imposed strain amplitude. Variations of $\theta_{\mathrm{s}}$ showed characteristic behavior; at a small imposed strain amplitude, the magnitude of $\theta_{\text {s }}$ gradually increased at an initial stage of fatigue process and showed an equilibrium value up to fatigue failure. On the other hand, at a large imposed strain amplitude, the magnitude of $\theta_{\mathrm{s}}$ increased at the initial stage of fatigue process with maximum excursion of $\theta_{\mathrm{s}}$.

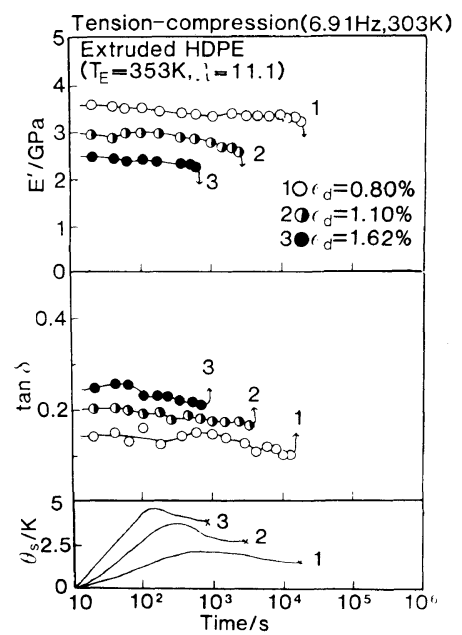

Figure 8. Variation of dynamic storage modulus, $E^{\prime}$, mechanical loss tangent, $\tan \delta$ and surface temperature, $\theta_{\mathrm{s}}$ for the extruded HDPE with time during the fatigue process under tension-compression type fatigue.

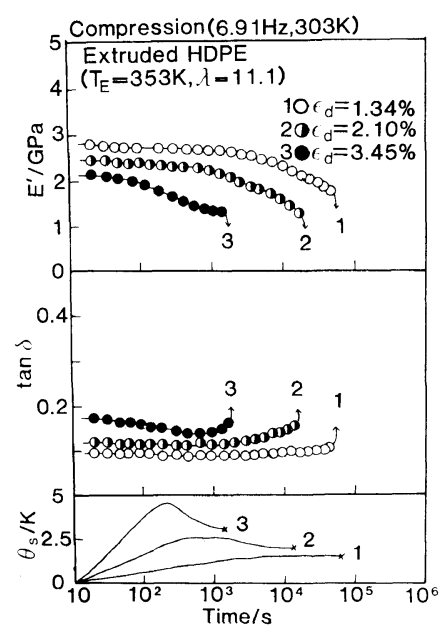

Figure 9. Variation of dynamic storage modulus, $E^{\prime}$, mechanical loss tangent, $\tan \delta$ and surface temperature, $\theta_{\mathrm{s}}$ for the extruded HDPE with time during fatigue process under compression type fatigue.

In the case of compression type fatigue (Figure 9), the magnitude of $E^{\prime}$ gradually decreased at the initial stage of fatigue process and then, showed drastic decrease after a certain fatigue time. The drastic decrease of the magnitude of $E^{\prime}$ was due to formation of a kink-bank corresponding to the appearance of 
maximum of $\theta_{\mathrm{s}}$ due to the start of effective dissipation of cyclic strain energy. Since the pure compression component of cyclic straining did not effectively stretch molecular chains, the maximum of $E^{\prime}$ induced by local stretching of molecules was not observed during cyclic fatigue test.

\section{Fatigue Fracture Criterion Based on Hysteresis Loss}

The hysteresis loss for viscoelastic materials per unit volume and unit time under cyclic straining is given by eq 1 .

$$
H=\pi f \varepsilon_{\mathrm{d}}^{2} \tilde{E}^{\prime \prime}
$$

where $f, \varepsilon_{\mathrm{d}}$, and $\tilde{E}^{\prime \prime}$ are frequency, strain amplitude and dynamic loss modulus, respectively. Since the magnitude of the hysteresis loss energy was greater than the generated heat energy as estimated from surface temperature rise, it is reasonably supposed that not all of the hysteresis loss energy was converted to heat under cyclic straining. Then, the hysteresis loss energy evaluated from eq 1 was divided into two parts, the energy dissipated as a heat generation and that used for a structural change under cyclic straining.

If the fraction of $\chi$ from the total hysteresis loss energy is converted to heat, the heat generation, $Q_{+}$, is expressed as $\chi \pi f \varepsilon_{\mathrm{d}}^{2} \tilde{E}^{\prime \prime}$. On the other hand, if the heat transferred to the surroundings is in proportion to the difference between the surface and ambient temperatures, the heat transfer rate, $Q_{-}$, is expressed as $\kappa \theta_{\mathrm{s}}$, where $\kappa$ is the heat transfer coefficient. Surface temperature rise is then expressed as follows:

$$
\mathrm{d} \theta_{\mathrm{s}} / \mathrm{d} t=1 / \rho c\left(\chi \pi f \varepsilon_{\mathrm{d}}^{2} \tilde{E}^{\prime \prime}-\kappa \theta_{\mathrm{s}}\right)
$$

where $\rho$ and $c$ are density and heat capacity, respectively. Equation 3 was used to evaluate the constant value of $\kappa$ from variation of $\theta_{\mathrm{s}}$ after fatigue failure:

$$
\mathrm{d} \theta_{\mathrm{s}} / \mathrm{d} t=-1 / \rho c\left(\kappa \theta_{\mathrm{s}}\right)
$$

The magnitude of $\kappa$ was $44 \mathrm{kJm}^{-3} \mathrm{~K}^{-1} \mathrm{~s}^{-1}$.

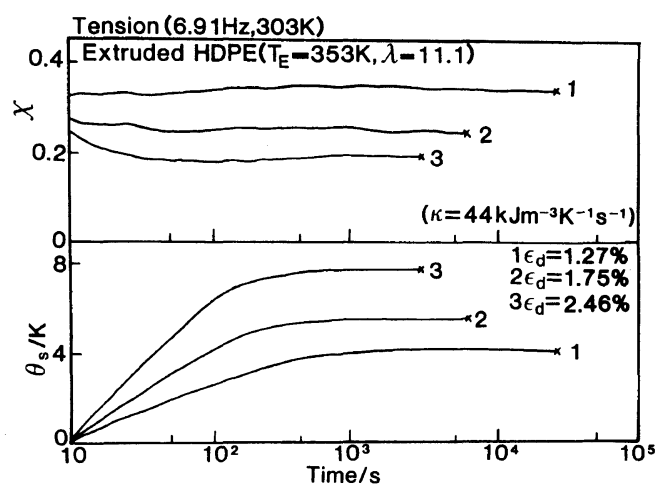

(a)

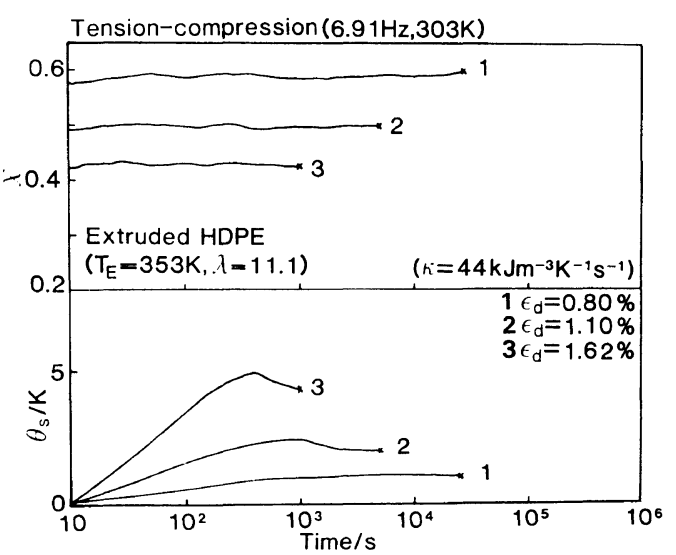

(b)

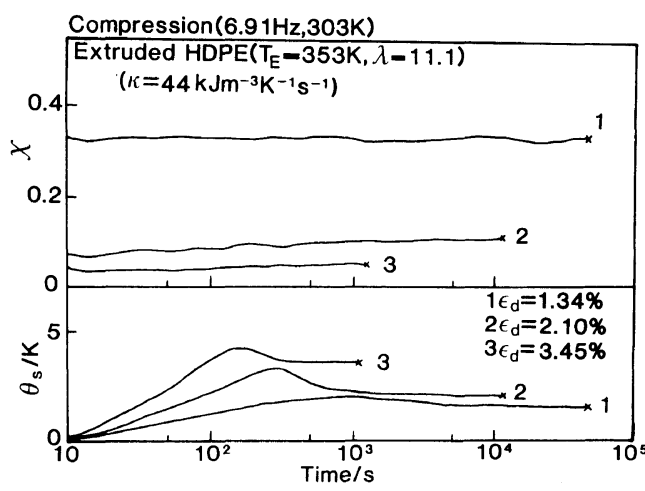

(c)

Figure 10. Variation of surface temperature rise, $\theta_{\mathrm{s}}$ and $\chi$ with time during fatigue process under various cyclic straining conditions. 
Figures 10(a)-(c) show variation of $\theta_{\mathrm{s}}$ and $\chi$ with time for the case of $\kappa=44 \mathrm{kJm}^{-3} \mathrm{~K}^{-1} \mathrm{~s}^{-1}$. The magnitude of $\chi$ was evaluated from curve fitting the right hand terms of eq 2 to the experimental values of $\mathrm{d} \theta_{\mathrm{s}} / \mathrm{d} t$ using fatigue time dependence. The magnitude of $\chi$ decreased with an increase of the magnitude of imposed strain amplitude. Since the fraction of $1-\chi$ might be mainly consumed by internal structural change during cyclic straining, it seems reasonable to consider that the magnitude of $1-\chi$ increased with strain amplitude, corresponding to a greater structural rearrangement. The magnitude of $\chi$ did not remarkably vary during cyclic fatigue process. In particular, in the vicinity of the maximum point of $\theta_{\mathrm{s}}$ observed under cyclic straining including compression component, no drastic change of $\chi$ was observed, even though kinkbands formed remarkably in this temperature region. This may be the reason why the formed kink-band was dispersed at local parts of the specimen.

In order to discuss the relationship between the magnitudes of $\chi$ and fatigue lifetime, the average value of $\chi$ during fatigue process, $\bar{\chi}$ was evaluated by the following equation.

$$
\bar{\chi}=\frac{1}{t_{\mathrm{f}}} \int_{0}^{t_{\mathrm{f}}} \chi \mathrm{d} t
$$

Figure 11 shows variation of $1-\bar{\chi}$ with fatigue lifetime under various straining conditions. The magnitude of $1-\bar{\chi}$ decreased with increasing fatigue lifetime. This clearly indicates that

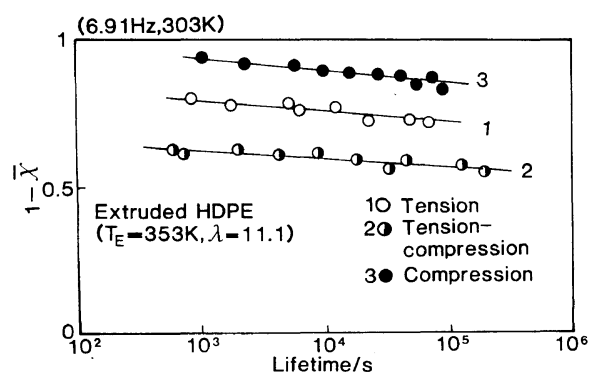

Figure 11. Relationship between $1-\bar{\chi}$ and fatigue lifetime, $t_{\mathrm{f}}$ for the extruded HDPE under various cyclic straining conditions. a reduction in the ratio of heat generation to the total hysteresis loss energy leads to shorter fatigue lifetime. Also, at the same level of $1-\bar{\chi}$, the magnitude of fatigue lifetime under compression type cyclic straining was longer than those of other cases, since generated crazes cannot easily propagate and grow into voids during the fatigue test.

The averaged hysteresis loss, $H_{\mathrm{av}}$ during cyclic fatigue was evaluated as follows:

$$
H_{\mathrm{av}}=\frac{1}{t_{\mathrm{f}}} \int_{0}^{t_{\mathrm{f}}}(1-\chi) H \mathrm{~d} t
$$

Figure 12 shows a plot of $H_{\mathrm{av}}$ against fatigue lifetime under various cyclic straining conditions. The slope of this logarithmic plot of $H_{\mathrm{av}} v s . t_{\mathrm{f}}$ is -1 in a short fatigue lifetime region. If a linear viscoelastic contribution is remarkable, for example, as in a case in which cyclic fatigue test with very small imposed straining and then, the fatigue test is carried out up to a range of extremely large lifetime, the fatigue limit hysteresis loss of $H_{0}$ should be evaluated as mentioned in the previous reports. ${ }^{6}$ In other words, the magnitude of $H_{\mathrm{av}}$ approaches a certain magnitude, $H_{0}$ in a fairly long fatigue lifetime region. Therefore, the empirical relationship between $H_{\mathrm{av}}$ and $t_{\mathrm{f}}$ can be represented by eq 6 as the fatigue criterion which is similar to the fatigue criterion proposed for unoriented polymeric specimens in the previous reports. ${ }^{5.6}$

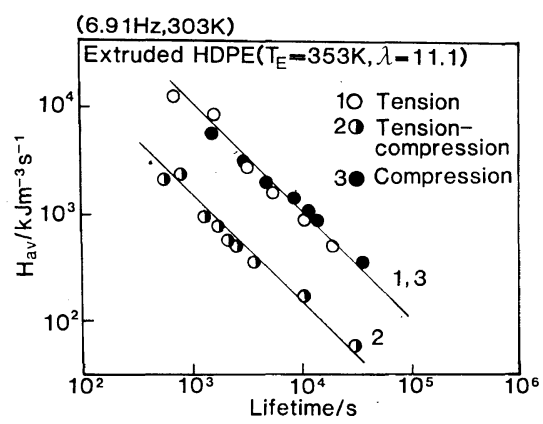

Figure 12. Relationship between average hysteresis loss, $H_{\mathrm{av}}$ and fatigue lifetime, $t_{\mathrm{f}}$ for extruded HDPE under various cyclic straining conditions. 


$$
\left(H_{\mathrm{av}}-H_{0}\right) t_{\mathrm{f}}=C
$$

where $C$ is total hysteresis loss up to a fatigue failure. Equation 6 means that fatigue failure occurs when the effective hysteresis loss energy, $\left(H_{\mathrm{av}}-H_{0}\right) t$ reaches a certain magnitude, $C$. At the same fatigue lifetime, $H_{\text {av }}$ for tensioncompression type cyclic straining is smaller than those for the other two types of cyclic straining. This indicates an ease of crack propagation due to kink-band formation. On the other hand, since crazes generated in the kink-band boundary are compressed, finally eliminating absorbing energy of cyclic straining in the compression type fatigue test, the fatigue lifetime is longer than that for tension-compression type fatigue compared with cases at the same magnitudes of $H_{\mathrm{av}}$.

\section{CONCLUSION}

Fatigue tests of highly oriented high density polyethylene (HDPE) were performed under tension-compression, pure tension and pure compression cyclic strainings. Morphological observations revealed that fatigue fracture behavior depended on the type of cyclic deformation. In the case of cyclic straining including a compression component, the fracture surface inclined by $45^{\circ}$ to the direction of cyclic deformation. On the other hand, in the case of tension type fatigue, the fracture surface was almost perpendicular to the direction of cyclic straining. The inclination of the fracture surface was in strong relation to kinkband formation along the direction of maximum shear stress.
From surface temperature rise and variation of dynamic viscoelasticity during fatigue process, a fatigue criterion based on hysteresis loss consumed for an internal structural change was established. A comparison of hysteresis loss energy consumed for a structural change for three different types of cyclic straining revealed that the fatigue strength in the tension-compression fatigue test is lower than those for the other two cases, since kink-band generated under compression cycle was extended under tension cycle.

\section{REFERENCES}

1. A. Cifferi and I. M. Ward, "Uitra-High Modulus Polymers," Applied Science, London, 1979.

2. M. N. Ridell, G. P. Koo, and J. L. O'Toole, Polym. Eng. Sci., 6, 363 (1966).

3. G. P. Koo, M. N. Ridell, and J. L. O'Toole, Polym. Eng. Sci., 7, 182 (1967).

4. I. Constable, D. J. Burns, and J. G. Williams, J. Mech. Eng. Sci., 12, 20 (1970).

5. A. Takahara, K. Yamada, T. Kajiyama, and M. Takayanagi, J. Appl. Polym. Sci., 26, 1085 (1981).

6. A. Takahara, K. Yamada, T. Kajiyama, and M. Takayanagi, J. Appl. Polym. Sci., 25, 597 (1980).

7. A. Takahara, K. Yamada, T. Kajiyama, and M. Takayanagi, J. Mater. Sci., 15, 2635 (1980).

8. N. Kaiya, M. Kugishima, A. Takahara, and T. Kajiyama, J. Soc. Fiber Sci. \& Tech., Jpn., 42, T-127 (1986).

9. R. E. Robertson, J. Polym. Sci. A-2, 9, 1255 (1971).

10. M. Takayanagi and T. Kajiyama, J. Macromol. Sci.Phys., B8, 1 (1973).

11. K. Shigematsu, K. Imada, and M. Takayanagi, $J$. Polym. Sci., Polym. Phys. Ed., 13, 73 (1975).

12. K. Imada, T. Yamamoto, K. Shigematsu, and M. Takayanagi, J. Mater. Sci., 6, 537 (1971).

13. M. Takayanagi and T. Matsuo, J. Macromol. Sci.Phys., B1, 407 (1967).

14. T. Kajiyama, T. Okada, A. Sakoda, and $\mathbf{M}$. Takayanagi, J. Macromol. Sci.-Phys., B7, 583 (1973). 